\title{
Response to the 2009 influenza A(H1N1) pandemic in Italy
}

C Rizzo (caterina.rizzo@iss.it) ${ }^{1}$, M C Rota $^{1}$, A Bella ${ }^{1}$, S Giannitelli ${ }^{1}$, S De Santis ${ }^{1}$, G Nacca ${ }^{1}$ M G Pompa ${ }^{2}$, L Vellucci ${ }^{2}$, S Salmaso ${ }^{1}$, $S$ Declich ${ }^{1}$

1. National Centre for Epidemiology, Surveillance and Health Promotion, Istituto Superiore di Sanità (ISS, National Institute of Health), Rome, Italy

2. Department of Prevention and Communication, Ministry of Health, Rome, Italy

Rizzo C, Rota MC, Bella A, Giannitelli S, De Santis S, Nacca G, Pompa MG, Vellucci L, Salmaso S, Declich S. Response to the 2009 influenza A(H1N1) pandemic in Italy. Euro Surveill. 2010;15(49):pii=19744. Available online: http://www.eurosurveillance.org/ViewArticle.aspx?Articleld=19744

Article published on 9 December 2010

In Italy, the arrival of the 2009 pandemic influenza $A\left(H_{1} N_{1}\right)$ virus triggered an integrated response that was mainly based on the 2006 National Pandemic Preparedness and Response Plan. In this article we analyse the main activities implemented for epidemiological surveillance, containment and mitigation of the pandemic influenza and the lesson learned from this experience. Overall, from week 31 (27 July - 2 August) of 2009 to week 17 (26 April - 2 May) of 2010, we estimate that there were approximately $5,600,000$ cases of influenza-like illness (ILI) who received medical attention (with almost 2,000 laboratory-confirmed cases of pandemic influenza from May to October 2009). A total of 1,106 confirmed cases were admitted to hospital for serious conditions, of whom 532 were admitted to intensive care units. There were 260 reported deaths due to pandemic influenza. Approximately 870,000 first doses of the pandemic vaccine were administered, representing a vaccine coverage of $4 \%$ of the target population. One of the possible reasons for the low uptake of the pandemic vaccine in the target population could be the communication strategy adopted, for both the general population and healthcare workers, which turned out to be a major challenge. Active involvement of all health professionals (at local, regional and national level) in influenza pandemic preparedness and response should be encouraged in the future.

\section{Background}

Since the emergence of the avian influenza threat in 1999, the Italian Ministry of Health in collaboration with the Istituto Superiore di Sanità, the national institute of health, started to work on an influenza pandemic preparedness plan. The first National Pandemic Plan for Preparedness and Response was developed in 2003 and subsequently updated in 2006 [1] according to the 2005 recommendations of the World Health Organization (WHO) [2]. The 2006 Plan was aimed at strengthening preparedness and response for an influenza pandemic at both national and local level by improving epidemiological and virological surveillance (identification, confirmation and timely reporting of cases), implementing containment measures at the early stage of a pandemic (e.g. border restrictions, isolation of the first possible, probable and confirmed cases, contact tracing), reducing the impact of the pandemic through the implementation of mitigation measures (pharmaceutical and non-pharmaceutical), ensuring communication strategies to inform healthcare workers, the media and public about decisions, and monitoring the efficiency of the interventions undertaken.

Since 2001, the National Health System has been decentralised and the 21 Italian regions are responsible for organising and delivering health services according to the Ministry of Health recommendations, including the necessary actions to contain and mitigate a pandemic. Each region was requested to produce its own Regional Pandemic Preparedness and Response Plan. This report summarises the response to the 2009 pandemic influenza $A\left(\mathrm{H}_{1} \mathrm{~N}_{1}\right)$ in Italy and the lessons learned from this experience.

\section{Initial response strategies}

After the first pandemic influenza alert was announced by WHO in late April 2009 [3], a National Crisis Management Committee, headed by the Minister of Health was established, in charge of coordinating the strategies related to preparedness, response and communication during the pandemic.

\section{Enhanced surveillance and data collection}

Seasonal Influenza surveillance is based on a nationwide sentinel surveillance network (INFLUNET) combining clinical and virological information. The system is based on sentinel practitioners (general practitioners and paediatricians) covering about $1.5-2 \%$ of the general population, with the aim of monitoring the incidence of medically attended influenza-like illness (ILI), identifying the extent of the seasonal epidemics and collecting information on circulating viral strains from week 42 to week 17 of the following year each influenza season. A case of medically attended ILI is defined as a patient attending a sentinel practitioner 
with acute onset of fever $>38^{\circ} \mathrm{C}$, respiratory symptoms and one of following symptoms: headache, general discomfort or asthenia. Data collected through INFLUNET are also uploaded weekly into the European Influenza Surveillance Network (EISN) database coordinated by the European Centre for Disease Prevention and Control (ECDC) [4].

Immediately after its formation, the National Crisis Management Committee recommended enhancing INFLUNET surveillance, so that it start earlier than usual in order to detect any sudden increase in the number of ILI cases in the community. The committee also decided that an active surveillance system should be set up to detect individuals presenting with ILI with a recent history of travel to the affected areas (Mexico and United States), as well as their close contacts. As previously described [5], individuals coming from affected areas received specific medical advice through the health authorities at airports and seaports to go immediately to a hospital if they developed symptoms of ILI. Any possible, probable or confirmed case of pandemic influenza - defined according to the European Union case definitions [6] - was immediately reported to the Ministry of Health. Moreover, laboratory confirmation of all suspected cases was required. Demographic data and information about symptoms and travel history were collected.

The first 200 confirmed cases of pandemic influenza were thoroughly investigated by local health authorities, using specific online epidemiological investigation forms, within 12 hours after case confirmation. Follow-up information was requested by the local health authorities for each case after 15 days. Data on contacts were also collected including exposure data (e.g. relationship to case, type and date of contact, household information) and subsequent development of illness and/or asymptomatic infection.

\section{Containment measures implemented}

Containment measures were implemented in April 2009 and included social distancing measures (early isolation of cases and precautionary closure of schools with more than five ILI cases with at least two confirmed) and antiviral prophylaxis for close contacts of cases. A stockpile of 40 million doses of antiviral drugs (sufficient for a complete treatment for approximately $4 \%$ of the whole population) stored by Ministry of Health was distributed to the regions, together with recommendations for their correct use [7]. Any person reporting to have been in close contact with a confirmed case was asked to remain at home for seven to 10 days, thus avoiding contact with others. This recommendation was maintained until the end of July 2009.

\section{Modelling disease spread}

As soon as the pandemic threat emerged, it was crucial for national policymakers to have early predictions on the possible spread of the pandemic virus. Since the early phase of the epidemic in Italy, real-time analysis was undertaken to provide weekly advice, together with epidemiological data, to the National Crisis Management Committee. Since the National Health Authorities request relevant information to tailor containment and mitigation measures to be implemented in the population and to understand the possible scenarios of the pandemic influenza burden in case of disease spread at the national level, a reference scenario on the spatio-temporal spread of the pandemic virus was provided, using mathematical modelling, and the effectiveness of mitigation measures, both pharmaceutical and non-pharmaceutical (such as school closure and social distancing measures), was assessed. Briefly, a stochastic, spatially explicit, individualbased simulation model was used. Individuals are explicitly represented and can transmit the infection to household members, to school or work colleagues and in the general population (where the force of infection is assumed to depend explicitly on geographical distance). The national transmission model was coupled with a global homogeneous mixing Susceptible Exposed Infected Removed (SEIR) model accounting for the worldwide pandemic, which was used for determining the number of cases imported over time. The transmission model used was parameterised, based on the existing evidence, derived from the analysis of data from the national surveillance system until 17 June 2009 and on estimates of key epidemiological parameters available at that time [8].

\section{Fine-tuning surveillance}

On 11 June 2009, the WHO Director-General raised the pandemic level to level 6 [3]. In July 2009, WHO made changes in the reporting requirements for pandemic influenza, because of the worldwide spread of the disease [9]. The Italian Ministry of Health modified the previous requirements: regions were required to report weekly an aggregate number of probable, possible and confirmed cases, confirmed hospitalised cases and deaths due to pandemic influenza [8].

In addition, the following pre-existing surveillance systems were expanded.

- A web-based emergency room hospital admissions and hospitalisations sentinel surveillance system had been in place since 2008. In August 2009, the system was enhanced, by increasing the number of emergency rooms surveyed. A network was established among Italian emergency services that had an automatic recording system for admissions. Of the 21 Italian regions, 12 identified at least one emergency service that would send data for surveillance; to date, these constitute the reporting units of the system. Data from the previous year, were used when available to estimate the number of weekly admissions. Epidemic thresholds were calculated using a Poisson regression model.

- A surveillance system of drug purchase - collecting data from a representative sample of 2,500 public and private pharmacies in Italy on the purchase of antibiotics (belonging to the Anatomical 
Therapeutic Chemical (ATC) Classification System (ATC J01), painkillers (ATC No2B) and antiviral drugs (ATC J05AH) - was incorporated into pandemic surveillance activities. All data refer to prescribed drugs except painkillers, which are also available in Italy over the counter. The system had been in place since January 2005.

In addition, the following surveillance systems were set up during the pandemic.

- A web-based data collection form for surveillance of severe confirmed hospitalised cases and deaths due to pandemic influenza was set up in midSeptember 2009. Forms were filled in by regional and local authorities and data were analysed daily at the national level (by the Istituto Superiore di Sanità and the Ministry of Health).

- To monitor vaccination coverage, in October 2009 a specific web-based data collection form was developed to be filled in by local health authorities (with details of the number of vaccine doses administered weekly to the target population, by age, risk conditions and region). Moreover, denominators for each target groups were also requested for each region in order to calculate vaccination coverage. The data were subsequently aggregated at the national level. Vaccination coverage reported always refers to the target population.

\section{Communication of data}

In order to inform the public about the pandemic in Italy and abroad, and to minimise conflicting information from different sources, communication to the public through the media was centralised at the national level and daily reports were published on the Ministry of Health website. When all surveillance activities were well established, a weekly report - including data and trends of ILI cases, vaccination coverage, emergency room admissions for acute respiratory syndromes, purchase of painkillers, antibiotics and antiviral drugs, and mortality - was released, in both Italian and English [10].

\section{Mitigation measures implemented}

Since 22 July 2009, the Ministry of Health recommended the use of antiviral drugs only for severe cases of pandemic influenza and for symptomatic patients with underlying medical conditions. In September 2009, the Ministry of Health started a health education campaign targeted at the general population recommending the adoption of basic non-pharmaceutical measures, such as staying at home if ill and covering noses or mouths with tissues, handkerchiefs or elbows when sneezing or coughing. Moreover, a specific hotline was set up to give advice and information regarding pandemic influenza prevention to both the general population and healthcare professionals.

Also in September 2009, according to the National Pandemic Preparedness and Response Plan before the pandemic vaccine became available, the Ministry of Health on 30 September 2009 identified the priority categories to be vaccinated, in a stepwise manner:

TABLE

Vaccination coverage for first dose of pandemic influenza vaccine by target group, Italy, October 2009 to May 2010

\begin{tabular}{|c|c|c|c|}
\hline Target groups & $\begin{array}{l}\text { Number of first doses } \\
\text { administered }\end{array}$ & $\begin{array}{l}\text { Number } \\
\text { of persons in target group }\end{array}$ & Vaccine coverage (\%) \\
\hline Healthcare personnel & 165,562 & $1,069,264$ & 15.5 \\
\hline $\begin{array}{l}\text { Essential services personnel (e.g. police, firefighters, } \\
\text { military corps) }\end{array}$ & 72,181 & $1,228,155$ & 5.9 \\
\hline Blood donors & 6,329 & 742,349 & 0.8 \\
\hline Pregnant women in their second and third trimesters & 23,016 & 189,915 & 12.1 \\
\hline $\begin{array}{l}\text { Women who delivered in the previous } 6 \text { months or person } \\
\text { who take cares of the baby }\end{array}$ & 8,170 & 237,594 & 3.4 \\
\hline $\begin{array}{l}\text { Individuals with at least one chronic underlying condition } \\
\text { aged } 6 \text { months }-65 \text { years }\end{array}$ & 549,167 & $4,309,466$ & 12.7 \\
\hline $\begin{array}{l}\text { Individuals with at least one chronic underlying condition } \\
\text { aged }>65 \text { years }\end{array}$ & 13,562 & 710,862 & 1.9 \\
\hline Children aged $>6$ months attending day-care centres & 4,618 & 89,394 & 5.2 \\
\hline Children aged $\ll 18$ years resident in long-term care facilities & 1,120 & 10,155 & 11.0 \\
\hline Children aged $<24$ months born pre-term & 1,595 & 20,657 & 7.7 \\
\hline Healthy children and adolescents aged 6 months -17 years & 20,307 & $7,671,581$ & 0.3 \\
\hline Healthy individuals aged $18-27$ years & 5,650 & $4,642,188$ & 0.1 \\
\hline Total & 871,277 & $20,921,580$ & 4.2 \\
\hline
\end{tabular}


1. healthcare personnel and essential services personnel (e.g. police, firefighters, military corps) including blood donors;

2. pregnant women in their second and third trimesters and women who delivered in the previous 6 months or persons who take care of the baby;

3. individuals with at least one chronic underlying condition aged 6 months -65 years putting them at high risk of severe or fatal complications due to pandemic influenza and children aged $<24$ months born pre-term;

4. children aged $>6$ months attending day-care centres

5. healthy children and adolescents (aged between 6 months and 17 years);

6. healthy individuals aged 18-27 years;

7. individuals with at least one chronic underlying condition aged $>65$ years.

The Table shows the vaccination coverage for the first dose of the pandemic vaccine during October 2009 to May 2010.

Agreements with pharmaceutical companies regarding the availability of pandemic vaccine according to the WHO indications [11] on the pandemic strain were signed by the Ministry of Health in 2005. On these bases and with the support of mathematical modelling showing that vaccinating $40 \%$ (24 million) of the Italian population (6o million) was adequate to mitigate the pandemic, the Ministry of Health decided to buy 24 million doses of adjuvated (MF59) vaccines from only one supplier. The selected company delivered half of the purchase to the Ministry of Health central storage from where vaccines have subsequently been distributed to the 21 Italian regions (since 12 October 2009) through the network of the Italian Red Cross.

\section{Evaluation of the pandemic in Italy}

Active surveillance of imported pandemic cases In Italy, the first imported confirmed case of pandemic influenza was detected on 24 April 2009 (week 17) [12]; by the end of July 2009 approximately 250 imported confirmed cases had been reported, with more than 2,000 suspected cases being investigated. In August 2009 the total number of medically attended ILI cases reached 5,000 , of whom approximately 2,000 (40\%) were laboratory confirmed. Since then the number of autochthonous clusters increased, suggesting sustained transmission in Italy, supported by the schools re-opening in mid-September. By mid-October 2009 (week 43) approximately 14,000 ILI cases had been reported.

INFLUNET sentinel surveillance system Even though the INFLUNET surveillance system had been in place from week 17 of 2009, no significant signals of increased influenza activity were detected until week 43, when an incidence of 4.5 cases per 1,000 served population of each reporting physician was observed. Two weeks later (week 45), the epidemic curve reached its peak, with a total incidence of 12.9 per 1,000 served population (Figure 1).

From week 31 of 2009 to week 17 of 2010, there were an estimated of approximately 5,600,000 medically attended ILI cases. The ILI incidence observed during the 2009-10 influenza season was 97 cases per 1,000 served population. This incidence estimate is similar to that described during the 2004-05 season, when the incidence rate reached the highest value ever described in Italy (116 cases per 1,000 served population). However, during the 2009-10 season, the number of ILI cases in the age group 0-14 years (270 cases per 1,000 served population) was the highest ever reported since the beginning of the INFLUNET surveillance system (which began in the 1999-2000 influenza season).

\section{FIGURE 1}

Incidence of influenza-like illness by age group, Italy, week 38 of 2009 to week 17 of 2010

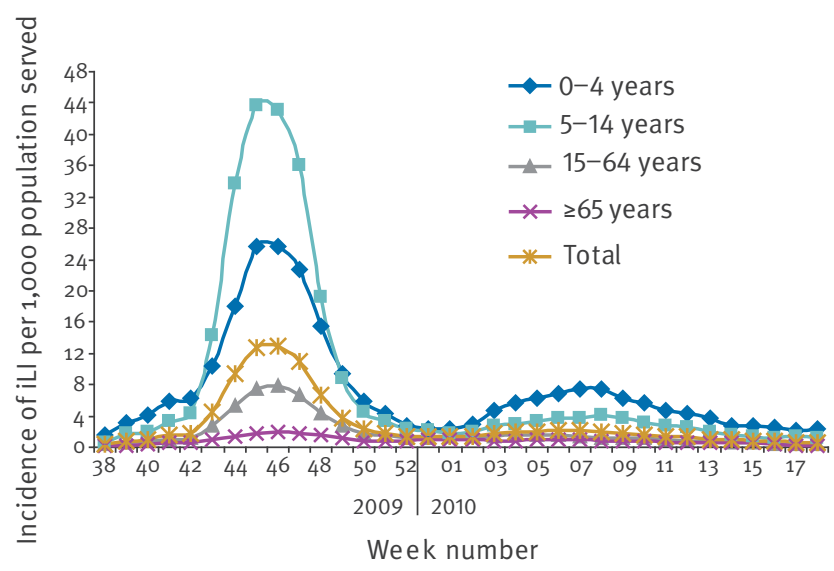

ILI: influenza-like illness.

Source: INFLUNET data.

\section{FIGURE 2}

Proportion of severe cases, admission to intensive care unit and deaths and incidence of influenza-like illness ${ }^{\text {a }}$, by age group, Italy

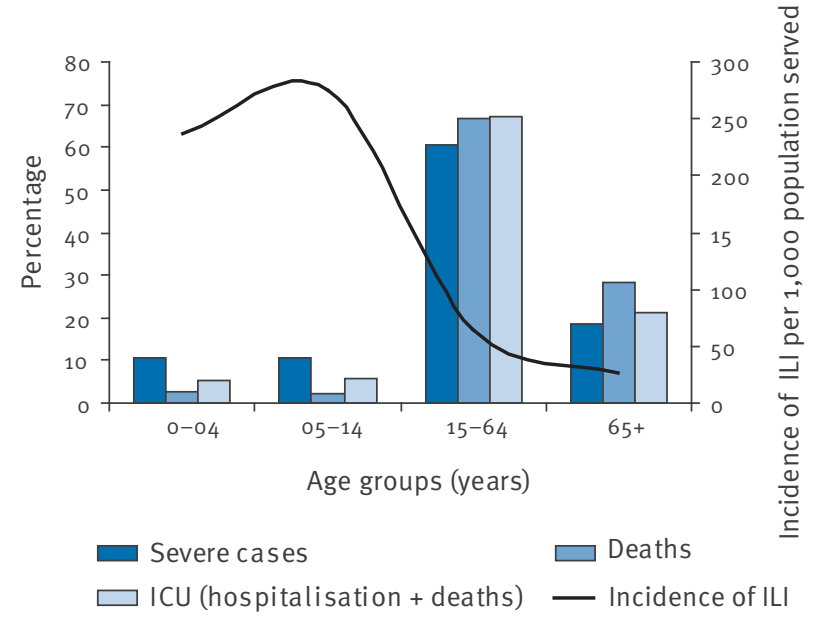

ICU: intencive care unit; ILI: influenza-like illness.

a Source: INFLUNET. 
In contrast, incidence in the age group $>64$ years was very low ( 26 cases per 1,000 served population).

\section{Surveillance of the first 200 confirmed pandemic influenza cases}

The epidemiological investigations of the first 200 confirmed pandemic influenza cases were collected using an online database established at the end of April 2009 after the first Italian laboratory confirmed imported pandemic influenza cases in the country. By the last week of October 2009, a total of 1,286 cases had been included in the database, with reported symptom onset dates from 24 April to 31 October 2009. Details of approximately 3,900 contacts were also included in the database. Most $(1,093$ of 1,$286 ; 85 \%)$ of the reported cases were notified by local health authorities within 12 hours after laboratory confirmation. Follow-up data were available for 1,040 of $1,286(81 \%)$ of the cases. In the later stage of the surveillance of the first 200 confirmed cases (end of September 2009 to November 2009), the proportion of cases that were followed-up decreased because the number of cases increased dramatically.

\section{Surveillance of laboratory- \\ confirmed severe cases}

Approximately 1,100 cases were admitted to hospital for serious conditions, of whom 532 were admitted to intensive care units, 49 needed extracorporeal membrane oxygenation, 166 were diagnosed with

\section{FIGURE 3}

Regions participating in the sentinel emergency room surveillance system, Italy, August 2009 to May 2010

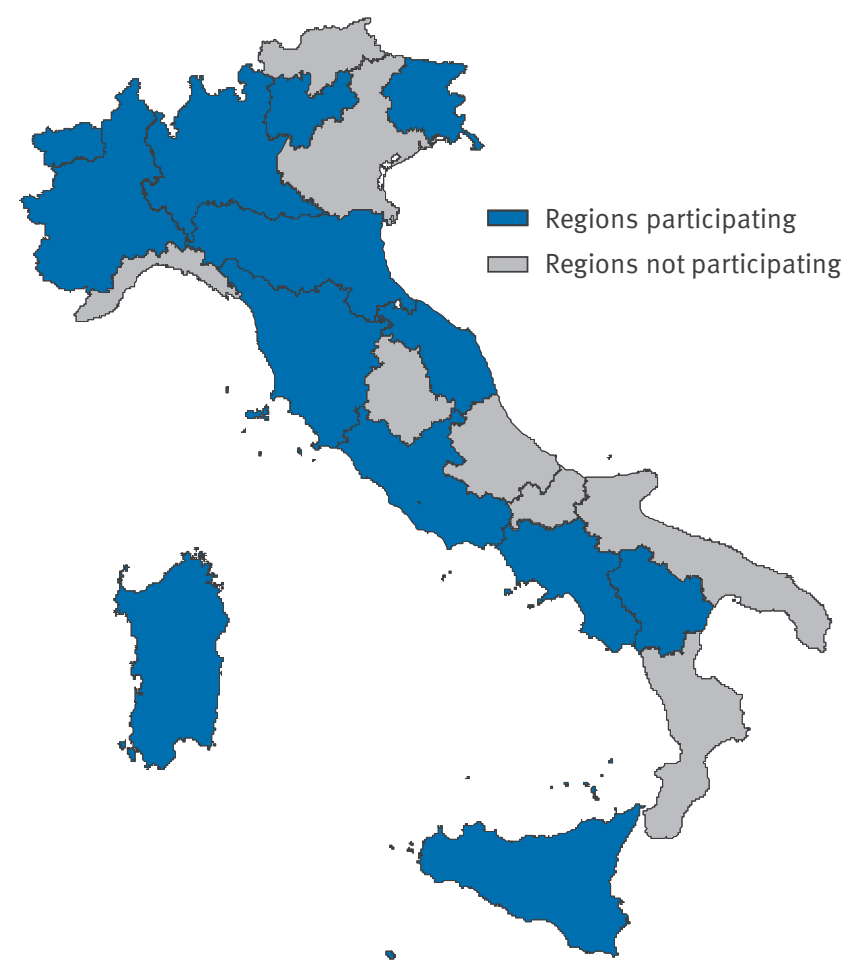

acute respiratory distress syndrome and 166 required oro-tracheal intubation. A total of 260 deaths due to complications arising from pandemic influenza were reported. In total, 476 of 1,100 (43\%) of hospitalised cases with available information were reported to have an underlying risk factor for severe disease, including pregnancy and obesity. Proportional distribution by age group of severe cases, number of cases who were admitted to an intensive care unit and number of deaths is shown in Figure 2. Data are compared with INFLUNET and clearly show that the incidence of ILI cases was higher in the children aged less than 14 years, while disease severity and fatal outcomes were concentrated in those aged over 15 years, with a mean of 43 years.

\section{Emergency room admissions}

The emergency room admission system collates data from 73 major, representative hospitals in 13 regions (Figure 3). Data reported during the week 43 of 2009 showed that $(3,269 / 43,335) 7.5 \%$ of all people who visited hospital emergency rooms were diagnosed with acute respiratory infection. Of these 653 (20\%), were admitted to hospital after being in an emergency room, with the baseline for admissions reached for the first time for all age groups. During week 45 of 2009, the peak was reached, with $12.2 \%$ of acute respiratory infection cases among emergency room visits $(4,995$ of 41,037); of these 863 (17.3\%) were hospitalised (Figure 4).

\section{Drug purchase}

A first peak in the purchase of antiviral drugs was registered in weeks 28 (6-12 June) to week 31 (July 27 to 2 August) of 2009, corresponding to the first pandemic wave registered in some northern European countries. In week 45, when the first peak of the ILI cases reported by INFLUNET in Italy was reached, a $90 \%$ increase in the purchase of antiviral drugs, and a $41 \%$ increase of antibiotics and a $95 \%$ increase of painkillers purchases were recorded, compared with the same week in 2008. Antiviral drug purchases reached 47 items per 100,000 inhabitants, more than double the amount bought the previous week, in line with the increase in the incidence of ILI.

\section{Mathematical modelling}

Simulations obtained by mathematical modelling were in agreement with the INFLUNET data in the early phase of the epidemic (April 2009 to September 2010), when containment measures were implemented. Briefly, by assuming isolation of confirmed cases, antiviral treatment and prophylaxis to $90 \%$ of symptomatic cases until 8 July 2009, and $33.3 \%$ natural immunity in the population aged more than 59 years, the peak of the ILI cases in Italy was expected on week 44 (95\% confidence interval: 44 to 45 ). Estimates were consistent with the INFLUNET data showing that the peak in Italy was reached in week 45-46 [8]. 


\section{Vaccine administration}

The pandemic vaccine was administered mostly by vaccination services; however, some regions also involved general practitioners and paediatricians in the pandemic vaccination campaign. Overall, 871,277 first doses and 52,723 second doses were administered (giving a total of 924,000 vaccine doses) and a national coverage among the target population of $4 \%$ (Table). Coverage was $15 \%$ of healthcare workers, $12 \%$ of pregnant women, $13 \%$ of persons aged under 65 years at high risk, and $11 \%$ of institutionalised individuals aged under 18 years old.

\section{Lessons learned}

When the pandemic virus emerged in late April 2009, reliable epidemiological data on the new circulating virus were limited and not available in a timely manner [13]. Consequently, uncertainty regarding the pathogenicity and severity of the pandemic virus, at the very beginning of its appearance, led advisors of decision-makers to consider the worst-case scenario. The combination of uncertainty and urgency to implement containment and mitigation measures in a short time made it difficult to fine-tune measures already included in the 2006 National Preparedness and Response Plan and to produce real-time modelling analysis with different scenarios of the possible impact of the mitigation measures. The WHO 11 June 2009 pandemic level 6 declaration supported the worst-case scenario approach. Therefore, on the basis of epidemiological data available in April 2009, only the actions listed in the 2006 Plan that were considered relevant to the situation at that time were performed. Among the activities undertaken, planning and coordination, situation monitoring and assessment, and containment and mitigation measures appeared to be efficient in the first containment phase (April- July 2009), in accordance with modelling results [8]. In fact, our experience suggests that the early response phase may have contributed to delaying and reducing the impact of the pandemic during spring and summer. This was facilitated also by school closure from early June to mid-September.

By contrast, the communication strategy adopted in Italy turned out to be a major problem. While at the beginning, the fast worldwide spread of the pandemic generated among the general population the feeling of a threat that was able to disrupt social life. Given the WHO pandemic level- 6 declaration in June 2009, it was quite clear that the 2009-10 pandemic was caused by a virus able to spread effectively between humans. The uncertainty of the data (regarding disease severity and real number of affected individuals and of deaths) between April and October 2009 caused a high degree

\section{FIGURE 4}

Influenza-like illness incidence and emergency room visits for acute respiratory infections, Italy, week 1 of 2009 to week 17 of 2010

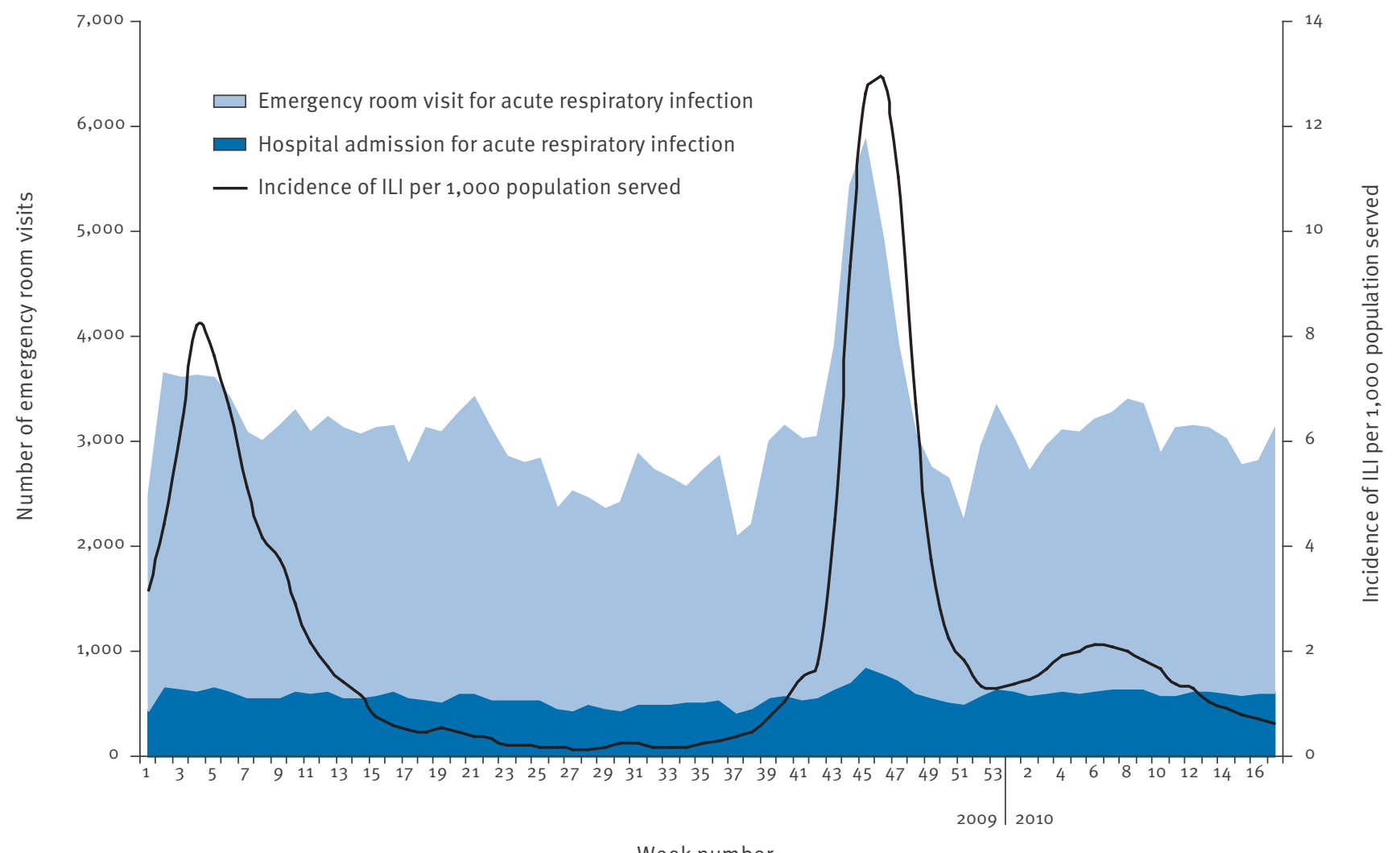


of disconcertion among healthcare workers and the public. This heavily influenced the vaccination campaign, in which the communication strategy plays a crucial role. The low vaccination uptake led to coverage of only $4 \%$ of the target population: $15 \%$ of the healthcare personnel and $1.5 \%$ of the general population [10].

In addition, the pandemic vaccines used during the 2009 pandemic were licensed by the European Medicines Agency (EMA) based on a mock-up vaccine procedure and were used on the basis of clinical data supporting the safety and effectiveness of vaccines developed using the influenza $A\left(\mathrm{H}_{5} \mathrm{~N}_{1}\right)$ strain, which had been thought would cause the next pandemic [14]. The way in which the pandemic vaccines were licensed was one of the main reasons of concern among healthcare workers and the general population. Another reason for concern was that this vaccine was a vaccine containing an adjuvant (MF59-squalene) and was recommended for risk groups (such as children and pregnant women) that differed from those included in the seasonal vaccination recommendations (elderly people and persons with underlying conditions older than 18 years) [15]. Concern was also raised by media regarding the risk of Guillain-Barré syndrome, related to the pandemic vaccine that was associated with 'swine influenza' vaccine that was administered in the United States in 1976-77 [16,17]. However, surveillance of adverse effect of pandemic influenza vaccination in Italy showed no particular evidence with respect to previous years [18].

These issues were mainly of concern to healthcare workers (e.g. general practitioners, paediatricians, specialists and nurses), who were supposed to liaise between the national and regional health authorities and the community. An Italian survey conducted in October 2009 among physicians and nurses, which investigated attitudes and behaviours towards preventive measures against the pandemic influenza, showed that: $70 \%$ of the 1,360 females (mainly nurses) in the sample and $51 \%$ of the 600 males would not get vaccinated against pandemic influenza [19].

Given this, many general practitioners and paediatricians were not able to disseminate the correct message, not even to the risk groups. Healthcare workers should have been timely informed about vaccine safety and involved in specific health education programmes in order to correctly inform the general population, but it was impossible to set up specific training before the end of December 2009, due to the overload of activities to be carried out during the pandemic. Indeed, concerns about vaccine safety should have been addressed first with general practitioners, using specific educational communication programmes. The fact that pandemic vaccine recommendations and prioritisation were based on risk rather than age strategies, coupled with the shortage of pandemic vaccines before the pandemic peak, vaccine dosage uncertainties, and the milder impact of the epidemic, concurred in discouraging the population to seek vaccination and probably had an important role in the failure of the vaccination campaign. This was the unfortunate consequence of the high level of uncertainties that informed most decisions during the period from July to September 2009.

As a result of the low vaccination coverage at national level, vaccine stock levels at the Ministry of Health warehouse remained high. In December 2009, a vaccine order was revised, 2,4 million doses were donated to WHO for developing countries, but the one-year validity of the vaccine doses forced the government to recall the doses and they will probably be discarded [20].

Enhanced epidemiological surveillance implemented in Italy during the pandemic substantially improved the quality and completeness of the epidemiological data collected. The integration of different data sources (i.e. incidence, mortality, severe cases, hospitalisation, emergency room visits, drugs purchases, pandemic vaccine coverage), allowed a weekly description of the burden of the 2009 pandemic influenza. This weekly epidemiological report (available also in English), disseminated through various official websites (Ministry of Health, Istituto Superiore di Sanità/National Centre for Epidemiology Surveillance and Health Promotion (Epicentro) and ECDC), has been a useful tool in informing and updating the media and health workers about the pandemic in Italy.

The intrinsic unpredictable characteristics of an influenza pandemic made every attempt of preparedness difficult and required flexibility in decision-making. However, the surveillance efforts made during this pandemic have provided a unique opportunity to validate influenza integrated surveillance, at both regional and national level. This surveillance, together with the established INFLUNET sentinel surveillance, will be maintained during the next influenza seasons. The underestimation of deaths could have been a weakness of the enhanced surveillance system adopted, because not all cases were laboratory confirmed.

The communication problems experienced during the pandemic also turned out to be valuable in generating a constructive discussion and building awareness of the importance of the active involvement of all health professionals (at local, regional and national level) in influenza pandemic preparedness.

In Italy responsibility for public health is shared between health authorities at national and regional level. Because of the threat posed by the pandemic, the regional health authorities implemented local pandemic plans. Thus, logistics issues, especially those concerning the distribution of vaccines within each region, as well as the strategy for the vaccinations at vaccination services or at the practices of general practitioners, were designed locally. Therefore, the response to the pandemic threat in Italy may have not been uniform and homogeneous, but it has strengthened the collaboration between central and peripheral levels. 


\section{References}

8. Italian Ministry of Health. National Plan for preparedness and response to an influenza pandemic. Rome: Ministry of Health. 2006. Available from: http://www.salute.gov.it/imgs/C_17_ pubblicazioni_511_allegato.pdf

9. World Health Organization (WHO). Pandemic influenza preparedness and response. A WHO guidance document. Geneva:WHO; Apr 2005. Available from: http:// www.who.int/csr/resources/publications/influenza/ WHO_CDS_CSR_GIP_2005_5/en/

10. World Health Organization (WHO). World now at the start of 2009 influenza pandemic. Statement to the press by WHO Director-General, Dr Margaret Chan. Geneva: WHO; 11 Jun 2009. Available from: http://www.who.int/mediacentre/news/ statements/2009/h1n1_pandemic_phase6_20090611/en/ index.html

11. European Centre for Disease Prevention and Control (ECDC). Weekly Influenza Surveillance Overview (WISO). Stockholm:ECDC; 2010. Available from http://ecdc.europa.eu/ en/healthtopics/seasonal_influenza/epidemiological_data/ Pages/Weekly_Influenza_Surveillance_Overview.aspx

12. Rizzo C, Declich S, Bella A, Caporali MG, Lana S, Pompa MG, Vellucci L, Salmaso S. Enhanced epidemiological surveillance of influenza $A\left(\mathrm{H}_{1} \mathrm{~N}_{1}\right) \mathrm{v}$ in Italy. Euro Surveill. 2009;14(27): pii=19266. Available from: http://www. eurosurveillance.org/ViewArticle.aspx?Articleld=19266

13. European Commission. Common case definition on novel flu virus. Brussels:European Commission; 30 Apr 2009. Available from: http://europa.eu/rapid/pressReleasesAction.do?referenc $\mathrm{e}=I \mathrm{P} / 09 / 690 \&$ format $=H T M L$

14. Italian Ministry of Health. Ordinanze e Circolari Nuova Influenza [Recommendations 2009 Influenza Pandemic]. Rome:Ministry of Health. 2009. http:// www.nuovainfluenza.salute.gov.it/nuovainfluenza/ archivioOrdinanzeCircolariNuovalnfluenza.jsp

15. Ajelli M, Merler S, Pugliese A, Rizzo C. Model predictions and evaluation of possible control strategies for the $2009 \mathrm{~A} / \mathrm{H}_{1} \mathrm{~N}_{1} \mathrm{~V}$ influenza pandemic in Italy. Epidemiol Infect. 2010;1-12.

16. World Health Organization (WHO). Changes in reporting requirements for pandemic $\left(\mathrm{H}_{1} \mathrm{~N}_{1}\right) 2009$ virus infection. Pandemic (H1N1) 2009 briefing note 3 (revised). Geneva:WHO; 16 Jul 2009. Available from: http://www.who.int/csr/disease/ swineflu/notes/h1n1_surveillance_20090710/en/index

17. National Centre of Epidemiology-Italian National Institute of Health (Cnesps-ISS). EpiCentro. FLUNEWS. Rome:Cnesps-ISS. [Accessed: 17 Apr 10]. Available from: http://www.epicentro. iss.it/focus/h1n1/archivioflunews.asp

18. World Health Organization (WHO). Availability of a candidate reassortant vaccine virus for the novel influenza $A\left(\mathrm{H}_{1} \mathrm{~N}_{1}\right)$ vaccine development CBER-RG2. . Geneva:WHO; 19 Jun 2009. Available from: http://www.who.int/csr/resources/ publications/swineflu/availability_candidate_reassortant_ vaccine_virus_CBER_RG2.pdf

19. Surveillance Group for New Influenza $A\left(\mathrm{H}_{1} \mathrm{~N}_{1}\right)$ Virus Investigation in Italy. Virological surveillance of human cases of influenza $A\left(\mathrm{H}_{1} \mathrm{~N}_{1}\right) v$ virus in Italy: preliminary results. Euro Surveill. 2009;14(24):pii=19247. Available from: http://www. eurosurveillance.org/ViewArticle.aspx?Articleld=19247

20. Ormsby CE, Reyes-Teran G. Influenza: making privileged data public. Science 2009;325(5944):1072-3.

21. European Medicines Agency (EMEA). Committee for Human Medicinal Products (CHMP). Guidelines on influenza vaccines prepared from viruses with the potential to cause a pandemic and intended for use outside of the core dossier context. London: EMEA; 24 Jan 2007. Available from: http://www. ema.europa.eu/docs/en_GB/document_library/Scientific guideline/2009/09/WC500003872.pdf

22. Italian Ministry of Health. Circolare per la prevenzione e il controllo dell'epidemia stagionale d'influenza 20092010. [Recommendations for prevention and control of seasonal influenza 2009-2010].Rome: Ministry of Health; 2009. Available from: http://www.salute.gov.it/dettaglio/ phPrimoPianoNew.jsp?id=250

23. Juurlink DN, Stukel TA, Kwong J, Kopp A, McGeer A, Upshur $R E$, et al. Guillain-Barre syndrome after influenza vaccination in adults: a population-based study. Arch Intern Med. 2006;166(20):2217-21.

24. Safranek TJ, Lawrence DN, Kurland LT, Culver DH, Wiederholt WC, Hayner NS, et al. Reassessment of the association between Guillain-Barre syndrome and receipt of swine influenza vaccine in 1976-1977: results of a two-state study. Expert Neurology Group. Am J Epidemiol. 1991;133(9):940-51.
25. Agenzia Italiana del Farmaco. A/H1N1 - Dati sulle sospette reazioni avverse al vaccino al 21 febbraio 2010. [A/H1 $\mathrm{N}_{1}$ - Data on suspected adverse events to the vaccine].Rome: Agenzia Italiana del Farmaco; 24 Feb 2010. Available from http://www. agenziafarmaco.it/it/content/ah1n1-\%E2\%80\%93-dati-sullesospette-reazioni-avverse-al-vaccino-al-21-febbraio-2010

26. La Torre G, Di Thiene D, Cadeddu C, Ricciardi W, Boccia A. Behaviours regarding preventive measures against pandemic $\mathrm{H}_{1} \mathrm{~N}_{1}$ influenza among Italian healthcare workers, October 2009. Euro Surveill. 2009;14(49):pii=19432. Available from: http://www.eurosurveillance.org/ViewArticle. aspx?Articleld=19432

27. World Health Organization (WHO). Pandemic vaccine donations for the developing world. Statement by WHO Director-General, Dr Margaret Chan. Geneva:WHO; 18 Sep 2009. Available from: http://www.who.int/mediacentre/news/statements/2009/ pandemic_vaccine_donations_20090918/en/index.html 